\section{SK1-I is the limit in AML}

\section{By Lauren Martz, Staff Writer}

A paper published in Blood suggests that going upstream of the known cancer target sphingosine 1-phosphate could be a better approach than hitting site-1 protease (S1P) itself. By specifically hitting one of the two sphingosine kinase isoenzymes that produce S1P, researchers at Virginia Commonwealth University School of Medicine think they have a small molecule cancer lead-dubbed SK1-I-with broader effects than S1P inhibitors and more selective effects than compounds that inhibit both isoenzymes. ${ }^{1}$ The upshot could be a compound for acute myelogenous leukemia with increased efficacy and fewer off-target effects.

$\mathrm{S} 1 \mathrm{P}$ is a sphingolipid mediator involved in calcium metabolism, cytoskeletal reorganization, immune cell trafficking, cell growth, differentiation, motility, angiogenesis and survival. ${ }^{2}$ It is produced by a pair of sphingosine kinase isoenzymes-sphingosine kinase 1 (SphK1) and SphK2 - that have opposite functions. ${ }^{3}$

SphK1 promotes proliferation and survival by catalyzing a reaction that produces S1P and depletes levels of ceramide and sphingosine, which are proapoptotic precursors of S1P. Studies have shown that SphK1 is upregulated in cancer cells. ${ }^{4}$

In the Blood paper, Sarah Spiegel and colleagues synthesized $(2 R, 3 S, 4 E)$ - $N$-methyl-5-(4'-pentylphenyl)-2-aminopent-4-ene-1,3diol, called SK1-I, which they said is the first water-soluble inhibitor specific for SphK1. Spiegel is corresponding author on the paper and a professor of biochemistry, molecular biology and oncology at Virginia Commonwealth.

In human leukemia cells, SK1-I increased the ratio of ceramide to S1P, which resulted in decreased cell growth and increased apoptosis. SK1-I reduced tumor volume by about $50 \%$ in xenograft mice. Moreover, the compound induced apoptosis in leukemic blasts from two human acute myelogenous leukemia (AML) patients while sparing normal leukocytes.

The only published SphK inhibitors "inhibit both SphK1 and SphK2 and are very toxic," because they also are blocking SphK2, Spiegel told SciBX.

Nonspecific SphK inhibition is dangerous because it causes a deficiency in S1P, which has such a broad range of cellular effects. Knockout of both SphK isoforms in mice disrupted normal neurogenesis and angiogenesis and was embryonically lethal. ${ }^{5}$

A key to eliminating cross-reactivity with SphK2 was designing SK1-I such that the molecule does not bind to its target's ATP-binding pocket. The binding pocket is a well-conserved region of many protein kinases, including SphK1 and SphK2.

\section{Better than blocking S1P}

In addition to SK1-I's potential for fewer side effects than pan-SphK inhibitors, the compound could have improved efficacy compared with S1P antagonists.

According to the Blood paper's authors, their molecule decreases "pro-growth and survival S1P while simultaneously increasing its precursor, the pro-apoptotic ceramide. Hence, SK1-I integrates multiple molecular therapeutic targets in leukemia."

Roger Sabbadini, VP and CSO of Lpath Inc., told SciBX that SK1-I might also have the potential to overcome chemotherapy resistance. He noted that SphK1 can prevent the apoptotic effects of chemotherapy drugs and that levels of SphK1 indeed correlate with resistance to chemotherapy.

Lpath is developing Asonep, a monoclonal antibody against S1P that is in Phase I testing for cancer.

Although Sabbadini acknowledged the benefits of specifically targeting SphK1, he cautioned that blocking the enzyme might not be as effective as targeting its prosurvival product, S1P. One reason, he said, is that targeting a kinase inside the cell (SphK1) is more difficult than targeting the extracellular sphingolipid (S1P).

Asonep "binds to and neutralizes S1P in the extracellular environments," Sabbadini noted. In addition, he said, "the major problem with targeting SphK1 is that there are other ways to make S1P. Our approach doesn't care where the S1P comes from."

Thus, Sabbadini said a potential next step for SK1-I is to "evaluate extracellular levels of S1P to see how much of the burden is eliminated by inhibiting its production. This could be demonstrated in preclinical mouse models."

Spiegel countered that the antibody approach only inhibits extracellular S1P. Her lab and others have shown that internal S1P also promotes growth and inhibits death of cancer cells. "Our inhibitor, by blocking production of S1P, would block this internal action of S1P as well as its external actions," she told SciBX.

Olivier Cuvillier, head of the Sphingolipids \& Cancer Research Lab at the Institute of Pharmacology and Structural Biology of the Centre National de la Recherche Scientifique (CNRS), agreed. He said the benefits of the approach published in Blood are that both the intracellular and possibly extracellular effects of S1P production are blocked.

Among other next steps, Sabbadini said, "we need to know the 


\section{TARGETS \& MECHANISMS}

pharmacokinetics and pharmacodynamics, half-life, biodistribution and druggability," of SK1-I. The authors of the Blood paper "need to initially put this compound into rodents, then nonhuman primates to establish a therapeutic index."

"We have already shown it can be administered to mice at effective doses without any obvious toxic effects," said Speigel. "After more complete toxicology and pharmacokinetics data, we hope to make modifications to the structure that will increase its potency."

Structure improvements could be challenging, noted Cuvillier, because "the major problem of the field is that the $3 \mathrm{D}$ structure of the kinase is not described. Until this is achieved, no really specific inhibitors will be found."

Eventually, SK1-I could find a home in a cocktail of therapeutics for AML.

Lina Obeid, professor of medicine and of biochemistry and molecular biology at the Medical University of South Carolina, noted that cancers usually disrupt multiple parts of the pathways that regulate cell growth and development. Thus, she said, inhibitors of SphK1 have potential in combination with therapies targeting other parts of the sphingosine pathway, such as S1P.

\section{REFERENCES}

1. Paugh, B. et al. Blood; published online May 29, 2008; doi:10.1182/blood-2008-02-138958

Contact: Sarah Spiegel, Virginia Commonwealth University, Richmond, Va.

e-mail: sspiegel@vcu.edu

2. Zhao, Y. et al. J. Biol. Chem. 282, 14165-14177 (2007)

3. Maceyka, M. et al. J. Biol. Chem. 280, 37118-37129 (2005)

4. French, K. et al. Cancer Res. 63, 5962-5969 (2003)

5. Mizugishi, K. et al. Mol. Cell Biol. 25, 11113-11121 (2005)

6. Payne, S. et al. FEBS Lett. 531, 54-57 (2002)

\section{COMPANIES AND INSTITUTIONS MENTIONED}

Centre National de la Recherche Scientifique, Paris, France Lpath Inc. (OTCBB:LPTN), San Diego, Calif.

Medical University of South Carolina, Charleston, S.C.

Virginia Commonwealth University School of Medicine, Richmond, Va. 